\title{
Frontières
}

\section{Andro-lithe et pétrification des cadavres humains au XIX siècle}

\section{Gian Marco Vidor}

Volume 23, numéro 1, automne 2010

Enquêtes sur le cadavre : 1 . Fascination

URI : https://id.erudit.org/iderudit/1004025ar

DOI : https://doi.org/10.7202/1004025ar

Aller au sommaire du numéro

Éditeur(s)

Université du Québec à Montréal

ISSN

1916-0976 (numérique)

Découvrir la revue

Citer cet article

Vidor, G. M. (2010). Andro-lithe et pétrification des cadavres humains au XIX ${ }^{\mathrm{e}}$ siècle. Frontières, 23(1), 66-73. https://doi.org/10.7202/1004025ar d'utilisation que vous pouvez consulter en ligne.

https://apropos.erudit.org/fr/usagers/politique-dutilisation/ 


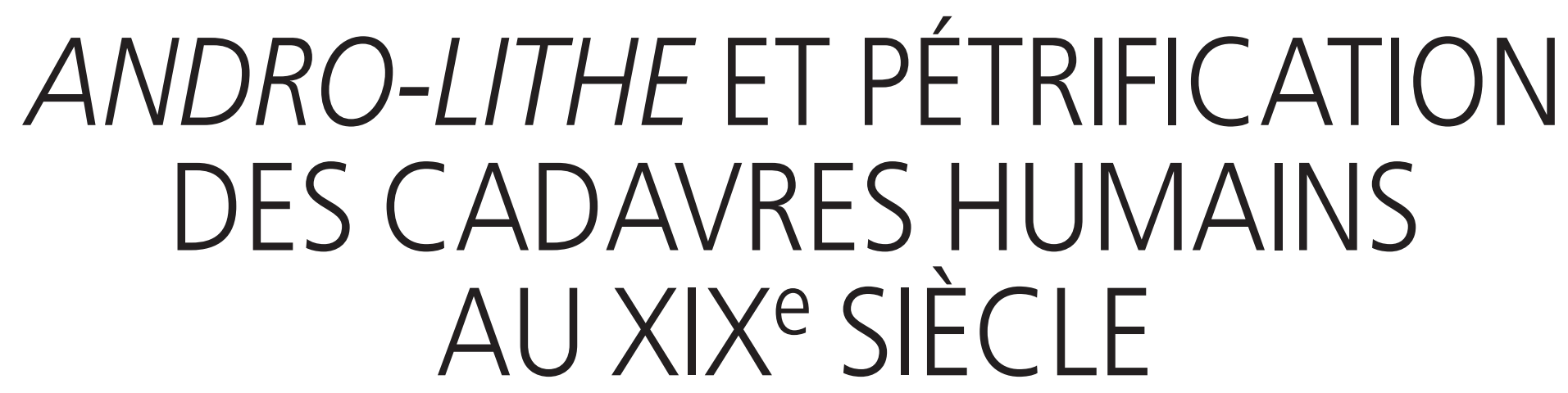

Gian Marco Vidor, Ph.D., chercheur, Institut Max-Planck, Berlin.

Dans le monde chrétien, on voyait dans le fait qu'un corps, en totalité ou dans ses parties, soit épargné du processus de putréfaction un signe visible de la sainteté des actions, des mots ou de la façon de mourir de l'individu auquel appartenait ce corps. Encouragée, approuvée ou simplement tolérée par l'Église, cette croyance dans l'intervention divine, transforme des restes humains plus au moins conservés en précieuses et puissantes reliques pour les fidèles (Bertley, 1985). Pourtant, la nature a toujours rendu imputrescibles des cadavres sans que personne n'y ait vu une intervention du ciel et les hommes ont, au cours des siècles, réussi de diverses façons à ralentir ou à arrêter la décomposition ou simplement à limiter les effets les plus intolérables de la mort sur le corps humain. Des documents écrits ainsi que des pièces anatomiques conservées dans de nombreuses institutions scientifiques italiennes et françaises permettent d'esquisser ce chapitre fascinant de l'histoire du corps humain.

Au Moyen Âge, ces interventions, généralement coûteuses, étaient réservées aux plus importants représentants du pouvoir religieux et du pouvoir temporel (Favole, 2003). Plusieurs procédés de conservation étaient pratiqués par différentes catégories de professionnels qui étaient appelées à intervenir sur les cadavres (du médecin au cuisinier). Souvent ces procédés prévoient l'éviscération, le lavage de la cavité abdominale, l'utilisation d'éléments simples comme le sel, des herbes, des épices, du miel et du vin ou bien des baumes coûteux, des substances rares, diverses par leur provenance, leur nature et leur composition. Ces pratiques d'embaumement - souvent des échecs - étaient indispensables pour garantir la conservation du corps pendant les longues cérémonies funèbres ou pour permettre le retour à la patrie de souverains, d'hommes d'Église ou de chevaliers morts ailleurs (Terribile Wiel Marin et Corrain, 1986; Grilletto, 1996). Dans certains cas, généralement restreints aux membres de la famille royale, de la cour ou de l'aristocratie, seulement certaines parties du corps faisaient l'objet d'une manipulation visant à les conserver: le cœur, les entrailles ou la tête étaient séparés du corps et envoyés pour être ensevelis séparément ailleurs (Ricci, 1998; Favole 2003). Même s'il est formellement interdit par l'Église, cet usage funèbre est pratiqué pendant des siècles.

Au Siècle des Lumières, grâce au perfectionnement des techniques de dessèchement des tissus et surtout de l'injection dans le système vasculaire des liquides, on parvient à bien préserver de la corruption des corps humains entiers, des organes, des systèmes, des membres qui étaient utilisés comme supports à la recherche et surtout à l'enseignement de l'anatomie humaine et comparée. Mais même dans le cas d'Honoré Fragonard (1732-1799) ${ }^{1}$, qui a dirigé le cabinet d'Anatomie comparée de l'École vétérinaire de Paris, à la fonction spécifiquement scientifique était toujours mêlée une dimension purement artistique: ces modèles étaient en quelque sorte des sculptures fantomatiques comme le fameux cheval et le chevalier attribués à Fragonard ${ }^{2}$. De tout autre nature et dimension était la collection d'un contemporain italien de Fragonard, Raimondo di Sangro (1710-1771) prince de San Severo. Alchimiste et franc-maçon, inventeur et savant qui parmi les résultats de ses expériences et recherches avait créé, avec l'aide d'un médecin, des macchine anatomiche $e^{3}$ : des corps desséchés constitués de la totalité de l'apparat vasculaire humain soutenu par le squelette. Dans la réalisation de ces machines, des motivations ésotériques, philosophiques et symboliques semblent avoir joué un rôle plus important que les intérêts purement scientifiques (Lista, 2005). Un grand précurseur de cet important chapitre de l'histoire de la conservation et de la mise en scène du corps humain est l'anatomiste hollandais 
Frederik Ruysch (1638-1731) dont le cabinet de curiosités avec ses sculptures complexes d'organes et ses corps embaumés était renommé dans toute l'Europe. Têtes couronnées, savants, académiciens et artistes faisaient de longs voyages pour les admirer. Et le tzar Nicolas le Grand, après les avoir vus deux fois, décida de les acheter pour l'université de Saint-Petersbourg après la mort de l'anatomiste (Grilletto, 1998; Mandressi, 2003).

Au cours du XVIII ${ }^{\mathrm{e}}$ siècle, avec la campagne d'Égypte de Napoléon, les savants d'Europe eurent la possibilité de voir et souvent d'analyser les anciennes momies qui à leurs yeux représentaient la perfection, désormais perdue, de l'art de l'embaumement. C'est au croisement entre égyptomanie et chimie que se place l'un des premiers hommes de science européens à avoir fait des expériences reconnues et documentées pour «réduire à la solidité de la pierre » les tissus animaux et humains, l'Italien Girolamo Segato (1792-1836). Né à Vedana, près de la ville de Belluno dans les Alpes centre-orientales, il part pour l'Égypte à l'âge de 20 ans, après ses études. Il y voyage jusqu'en 1823, faisant de la cartographie et du dessin. À son retour, il s'installe à Florence, alors capitale du progressiste Duché de Toscane. Malgré la perte d'une grande partie de ses documents lors d'un incendie et les difficultés économiques, il arriva à publier le premier tome d'un ouvrage sur l'Égypte. Mais la fuite de son associé et les dettes contractées pour ce projet de publication l'obligèrent à abandonner l'entreprise; c'est à ce moment-là que ses intérêts se tournèrent vers la pétrification des tissus. En 1833, Segato écrit à son frère qu'après plusieurs essais, il a réussi à «conserver des pièces d'anatomie humaine et comparée, comme le corps entier d'un animal avec ses entrailles, des mollusques et autres animaux formés de substance muqueuse» (cité par Grilletto, 1986, p. 52). Les académiciens, selon ce qu'écrit Segato, auraient montré un intérêt pour ses pièces après avoir examiné leur solidité, leur sécheresse et leur inaltérabilité à l'action de l'atmosphère, des mites et des insectes. Les professeurs d'anatomie et d'histoire naturelle auraient même souligné l'utilité didactique et les possibilités économiques de ses découvertes. En 1831, peu avant cette lettre, dans un court document publié dans la revue Antologia di Firenze (Segato, 1831, p. 74), Segato avait présenté au public les premiers résultats de ses expériences. Dans ce document, il ne parle pas de pétrification ou de réduction à l'état de pierre, mais il utilise, comme dans d'autres de ses écrits, le terme de «sécheresse». Ce sont les admirateurs de Segato, qui se rendent chez lui pour appré- cier et examiner sa collection, qui font référence à la «consistance et apparence de pierre» de ses «objets». Gaetano Mazzoni de Prato, connu pour ses études sur les insectes, écrit par exemple que Segato était capable de transformer la matière élastique du cadavre en pierre prête à être polie. Et Isabella Rossi décrivait son laboratoire comme étant plein de merveilleuses pétrifications (Pellegrini, 1836). Mais celui qui a contribué le plus à la renommée de Segato, in vita et post mortem, a été son grand ami, l'avocat Giuseppe Pellegrini. Avant de publier, en 1836, un ouvrage dédié à Segato, il avait envoyé un rapport à l'ancienne Società Medico-Chirurgica de Bologne, accompagné de déclarations écrites par les scientifiques les plus connus de Florence 4 . Pellegrini déclarait que Segato était capable de donner «la dureté de la pierre aux corps et aux membres de n'importe quel animal» (Pellegrini, 1836). Le processus de putréfaction était arrêté ou même prévenu, la couleur, la forme et les caractéristiques de la partie traitée ne changeaient pas, tout en restant complètement inodores. Les membres pouvaient même avoir une consistance qui les rendait inaltérables, tout en demeurant flexibles aux articulations. Les poils et les cheveux de ces pièces préparées restaient immuables et la peau pouvait même conserver les taches ou les couleurs liées à une maladie. Après le traitement, les oiseaux conser- vaient leurs plumes, les poissons leurs écailles, les insectes la plus délicate de leurs extrémités. Les squelettes pétrifiés étaient très solides et résistants à l'attaque des agents atmosphériques, de l'eau et des mites. Selon la description que Pellegrini faisait aux membres de la Società, la collection de Segato était composée, en plus des pièces d'origine animale, de bras, pieds et mains humains, d'organes comme le foi, le cerveau, les entrailles d'un enfant, etc. Mais les pièces les plus célèbres - et qui existent encore aujourd'hui - étaient la tête et le torse d'une jeune femme et la très connue "petite table marquetée».

Cette table ovale de $196 \mathrm{~cm}$ de circonférence est composée de 214 pièces anatomiques qui, en raison de leur couleur, de leur consistance et de leur luminosité ressemblaient - et ressemblent encore aujourd'hui - à des pierres dures ${ }^{5}$. On ne trouve cependant aucune référence à un corps humain entier. En effet, Segato n'a jamais pu obtenir la permission de traiter un corps humain entier dans son laboratoire probablement, comme le disent ses biographes, à cause des interventions de ses détracteurs au sein de l'Église et du gouvernement de Florence. Malgré le soutien d'une partie du monde académique et politique florentin, il n'arrivera pas à obtenir un poste stable dans les institutions de sa ville d'adoption; le bon accueil que recevront ses recherches à Bologne arrivera trop tard

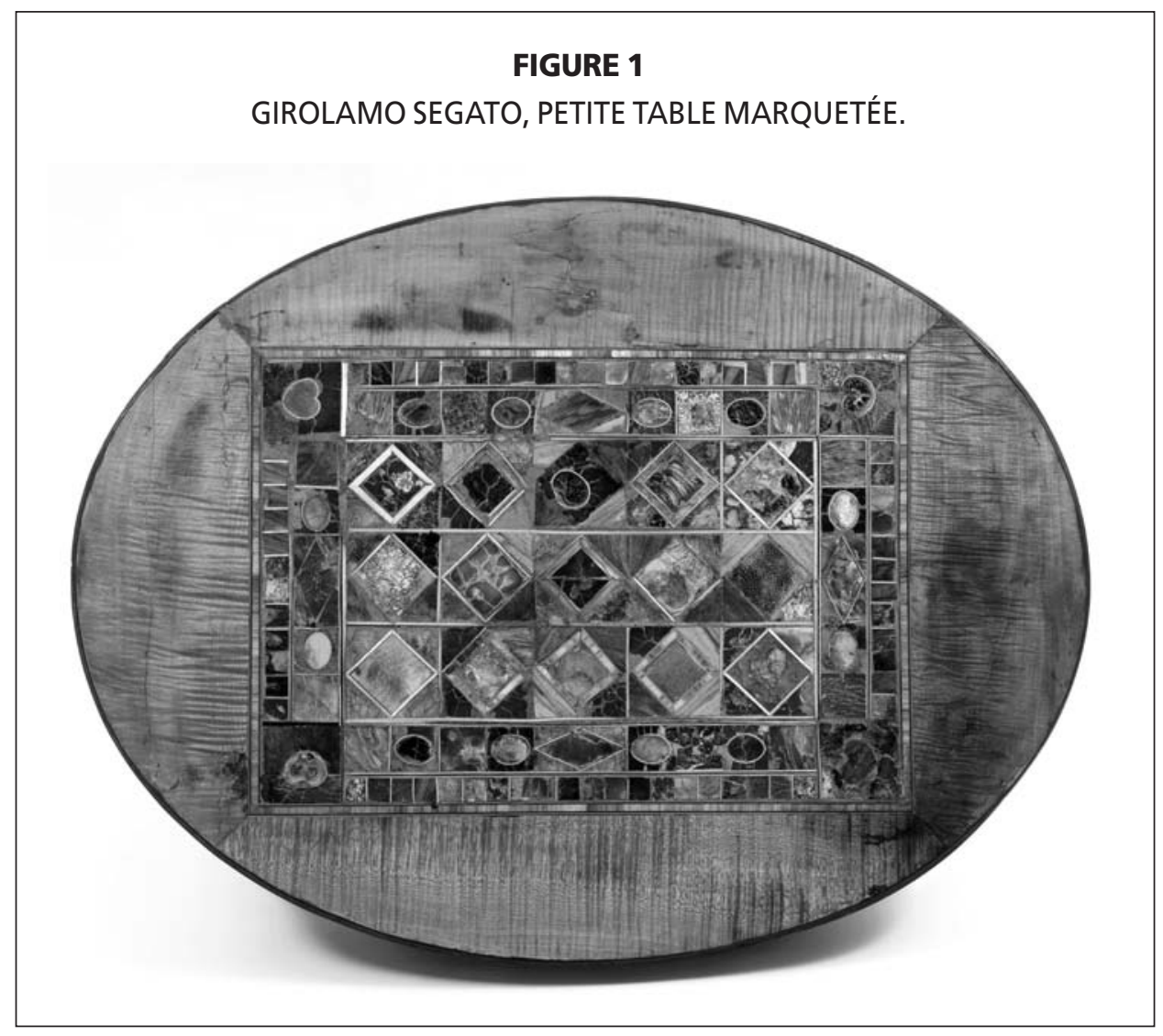

(C) Museo Galileo, Florence 
pour lui être utile, car il meurt l'année suivante, en 1836. Endetté, sans aucune position scientifique, académique et professionnelle reconnue, il décède alors que ses méthodes et ses formules sont encore en pleine phase d'amélioration, sans les avoir communiquées à qui que ce soit dans son entourage. La mort l'a empêché de compléter ses expériences et de partager ses découvertes «qu'il emportera avec lui dans la tombe». Accompagné par une foule importante, son corps sera enseveli dans l'église de Santa Croce à Florence ${ }^{6}$, où sera construit un monument funèbre payé grâce à une souscription publique organisée par ses admirateurs. On peut lire sur ce monument: "Ci-gît défait Girolamo Segato de Belluno qui pourrait être entièrement pétrifié si son art ne fuisse pas morte avec lui [...]». Sa mort prématurée, la non-divulgation de ses formules, sa vie retirée du monde et le fait qu'il a été mis à l'écart par le monde scientifique et médical de son époque ont fait de Segato plus un "alchimiste», un "magicien » qu'un représentant de la science du XIX ${ }^{\mathrm{e}}$ siècle.

Des analyses scientifiques récentes ont vérifié de plus près les caractéristiques des pièces anatomiques de Segato (Pocchiesa et Fornaro, 1992, p. 294-310). À côté des pièces qui, encore aujourd'hui, ont véritablement la consistance et l'apparence de la pierre, comme les morceaux qui composent la fameuse "petite table marquetée », il y en a d'autres qui sont ce que l'on pourrait appeler des «faux». Comme l'avait déjà constaté Guido Bustico (1896), le torse de la jeune femme est simplement la peau complète des seins, traitée et appliquée sur un modèle en plâtre, et les intestins "pétrifiés» sont en réalité des intestins remplis de plâtre. De plus, plusieurs de ces pièces que les contemporains de Segato décrivaient comme incorruptibles ont été au cours des deux siècles attaquées par les mites. Mais la découverte de ces petites astuces n'affaiblirent en rien sa renommée: Segato était l'homme de la pétrification.

Sans jamais l'avoir rencontré, Angelo Comi, professeur de chirurgie à Rome, est considéré par ses contemporains comme «le continuateur de l'art de Segato ${ }^{7}$. Les découvertes de ce professeur, membre de l'Accademia Pontificia dei Lincei, sont conservées presque essentiellement dans un ouvrage publié en 1839 par un professeur de Rome dont on connaît seulement les initiales D.G.R. Le document intitulé Intorno all'artificiale solidificazione lapidea, qui est presque une hagiographie, présente la biographie et les conquêtes scientifiques du jeune professeur Comi, âgé alors d'à peine 27 ans. Ce texte est complété par une vingtaine de documents (lettres, comptes rendus, déclarations et articles de presse) qui témoignent du succès qui entourait, dans la Rome de l'époque, les pétrifications d'Angelo Comi. Né à Rome en 1812, il entre très tôt à l'hôpital de S. Giacomo où il suit une formation en chirurgie. Selon son biographe, il commence alors ses expériences de conservation des tissus et des végétaux. En 1839, plusieurs chirurgiens, médecins et naturalistes italiens analysent les objets préparés par Comi et formulent leurs impressions dans des comptes rendus ou dans de simples déclarations (D.G.R. [s.n.], 1839). On pouvait voir le corps complet d'un Éthiopien dont les formes étaient parfaitement conservées - en particulier celles du visage - et dont la peau présentait une apparente fraîcheur. En outre, selon un groupe des chirurgiens de Rome, la méthode utilisée semblait garantir une conservation de plus longue durée par rapport aux «processus anatomiques connus jusqu'à ce moment» (D.G.R. [s.n.], 1839, p. 41). Dans leur compte rendu, ces chirurgiens parlent d'une "enveloppe cutanée» (D.G.R. [s.n.], 1839, p. 41-42) et non d'un corps complet (ce qui ferait penser à la présence des organes internes) comme le fait l'auteur de Intorno all'artificiale solidificazione lapidea. Dans ce document, on souligne la parfaite conservation des poils et des cheveux, des organes génitaux et surtout la grande flexibilité des articulations des bras et des jambes: "l'Éthiopien de Comi » pouvait être placé assis, avec les jambes croisées, ou debout, avec les mains levées, etc. La flexibilité de ce «petit Africain de 22 ans mort à Rome» avait impressionné aussi Gaetano Mazzoni, un voyageur toscan qui visite la collection du professeur Comi en 1839 (Mazzoni, 1938, p. 102). Comi a aussi produit une série considérable d'animaux, de plantes et quelques pièces anatomiques humaines parfaitement pétrifiées, même dans les parties plus délicates, en conservant leurs formes et leurs couleurs, comme le déclarent tous ceux qui ont pu les analyser. Les académiciens se réjouissent que l'art de Gerolamo Segato ait été non seulement poursuivi mais aussi amélioré par Angelo Comi.

Mais l'enthousiasme de D.G.R. et le soutien du milieu scientifique romain ne sont pas partagés par les membres de l'Académie de Sciences de Bologne, laquelle était à l'époque parmi les plus importantes institutions scientifiques italiennes et européennes. Angelo Comi avait envoyé à Bologne certaines de ses pièces pétrifiées (du sang humain, un testicule de coq, des poissons et beaucoup de fleurs). Les académiciens bolognais, dans un rapport publié dans les comptes rendus de l'Académie en 1840 , déplorent que la couleur originale ne soit pas conservée pour la plupart des pièces animales et le manque de parties importantes qui, pour les plantes, ont été remplacées par des éléments artificiels. Selon eux, les méthodes existantes, surtout en botanique, étaient plus efficaces que la méthode utilisée par le professeur Comi. Ce dernier aurait dû améliorer son processus, pour lequel l'Académie ne voyait pour le moment aucune réelle application ${ }^{8}$. L'état de la recherche sur l'œuvre d'Angelo Comi ne permet pas de savoir s'il a réussi à améliorer ses méthodes, mais son processus de pétrification est resté sans aucune application concrète.

À Paris, à la fin de la première moitié du XIX $^{\mathrm{e}}$ siècle, l'Académie de Médecine était occupée à juger diverses méthodes d'embaumement du corps humain présentées par ceux qui étaient les grands spécialistes de la matière, tels les docteurs Sucquet et Gannal (voir Poiseuille, Sucquet et Dupré, 1847). Selon ce qu'écrit Andrea Cozzi (1840) dans son ouvrage dédié à l'embaumement, Gannal aurait même travaillé sur des méthodes de pétrification qu'il aurait présentées au monde académique français. Mais cette information ne semble trouver aucune confirmation. Il faut souligner qu'en France, la question de la conservation du corps humain était alors d'une grande actualité mais était orientée plus vers la sphère du business funéraire que vers celle de l'anatomie et de la didactique médicale comme dans le cas italien. La pétrification n'était pas au centre des intérêts du milieu médical et scientifique français de l'époque dont les membres travaillaient non pour obtenir la solidité de la pierre mais pour une conservation du corps de durée moyenne et surtout pour le maintien de la couleur et des traits du visage et des mains du défunt, ce qui lui conférait une dimension d'endormi et non de cadavre.

Pendant la deuxième moitié du $\mathrm{XIX}^{\mathrm{e}}$ siècle, et en particulier en France, on produit une grande quantité de projets, d'études, de rapports, de propositions autour de sujets comme la mort, la gestion des morts, les cimetières. Hommes politiques, administrateurs, membres des académies, hommes d'affaires et petits fonctionnaires veulent contribuer à la grande aventure du cadavre à l'époque du triomphe de la science et de la technique. Chacun d'eux a son projet révolutionnaire, sa loi civilisatrice, sa découverte sensationnelle, son invention ingénieuse et utile. C'est dans ce grand marché de la mort bourgeoise de la deuxième partie du $\mathrm{XIX}^{\mathrm{e}}$ siècle que l'on retrouve deux projets liés, même si de façon très particulière et opposée, à la pétrification du corps humain: l'Androlithe de Urban et le nouveau mode de sépulture par la pétrification de Boitel. 
Ce dernier, agronome à GennevilliersAsnières, publie une brochure dans les années 1880 où il présente son invention, pour laquelle il dit avoir obtenu un brevet valable pour la France et à l'étranger (Boitel, s.d.). Rappelant à ses lecteurs que la nature opère la transformation en pierre des animaux et des plantes, il affirme avec beaucoup de satisfaction avoir réussi à solidifier les corps humains qui «se durcissent de manière à être changés en une espèce de ciment romain ou de brique imperméable qui résisterait à toutes les injures du temps » (Boitel, s.d., p. 3). Dans la partie de sa brochure portant sur un éventuel perfectionnement de son mode de pétrification, il admet un point faible: il n'arrivait pas à conserver les traits du visage dans «un degré de ressemblance reconnaissable».

Quel est ce nouveau mode de conservation des cadavres? Boitel le présente de manière très détaillée. Il consiste à placer le corps le plus rapidement possible à l'intérieur de ce qui, à la fin du processus, ressemble à un grand cocon de ciment.

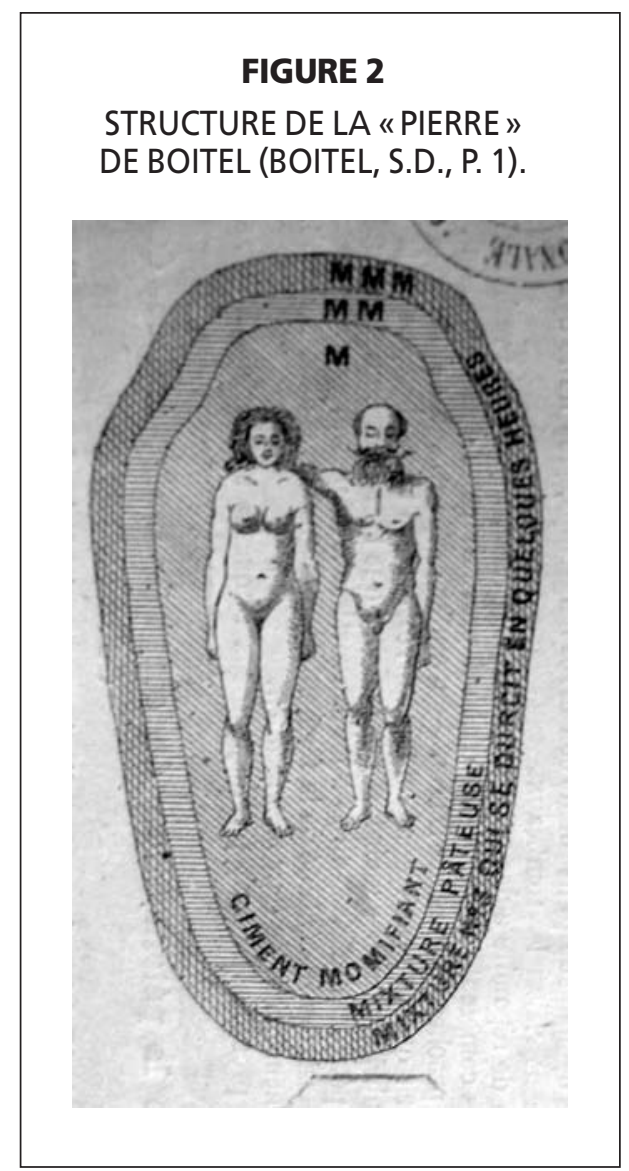

(C) Bibliothèque Nationale de France

Ce cocon est composé de trois couches distinctes de densité graduée. Le corps nu, sans vêtements ni suaire, est immergé dans la «mixture la plus riche, préparée à l'état sirupeux» (lettre M de la figure 2) qui, grâce à des processus chimico-physiques, doit amener la pétrification. Cette mixture est une solution astringente d'une densité différente de celle des liquides contenus à l'intérieur du cadavre. Il s'établit, dès lors, à travers la peau qui fait office de membrane poreuse un courant entre ces liquides et la solution. Celle-ci pénètre dans les tissus, les durcit et les ossifie, empreignant la peau des liquides filtrés qui se volatilisent peu à peu dans l'atmosphère (Boitel, s.d., p. 7).

La deuxième et la troisième couche ont été partiellement préparées à l'avance de façon à créer la moitié inférieure de ce qui sera le pseudo-rocher final. La deuxième couche, à l'état pâteux, achèvera la résorption des «aqueux cadavériques» (Boitel, s.d., p. 7). La troisième est «une sorte d'enveloppe extérieure cylindrique formée d'un enduit bitumineux déjà solidifié, mais assez poreux pour faciliter l'essor complet des liquides» (Boitel, s.d., p. 7). Cette troisième couche est en réalité la première à être réalisée, au moins dans sa partie inférieure. Elle constitue une sorte de «mortier», pour utiliser l'image de Boitel, obtenu à l'intérieur d'un «vaisseau portatif (soit une bière ordinaire, soit une auge improvisée, soit une baignoire) »(Boitel, s.d., p. 7), en mélangeant différents éléments indiqués par l'auteur. Les trois enveloppes vont progressivement constituer un tout homogène où le corps est enfermé.

Au moment des obsèques, selon Boitel, " on enlève du récipient ad hoc cette masse informe de concrétions. Le revêtement extérieur étant à ce moment déjà solide, on peut placer le tout dans une bière en bois ou en métal, ou le laisser à découvert. Dans l'un et l'autre cas la pétrification se continue d'elle-même à l'intérieur» (Boitel, s.d., p. 7). Ce bloc de pierre peut être immédiatement déposé dans un caveau funéraire, une chapelle, ou laissé en plein air comme un mausolée. Il peut être décoré: inscriptions funéraires, motifs honorifiques, emblèmes religieux; il peut aussi être taillé en statue ou recouvert de bronze. Selon notre inventeur, dans les petites villes un seul préposé, aidé d'un médecin, suffirait pour préparer l'ensemble; dans les plus grandes villes, les opérations seraient conduites par les services des pompes funèbres.

Sans spécifier la durée, Boitel affirme que le corps pouvait être libéré du bloc lorsqu'il se serait desséché. En effet, grâce sa méthode, on arrive à obtenir «à peu près les résultats des momies blanches ou arabes, cadavres enfouis dans les sables de l'Arabie et de la Lybie et desséchés par les ardeurs du soleil» (Boitel, s.d., p. 3). Cette phrase, à elle seule, montre comment en réalité le résultat de ce que Boitel présente comme un mode de pétrification n'est en fait que la simple déshydratation des parties molles, donnant au corps l'aspect d'une momie naturelle, ce qu'à l'époque on pouvait appeler «arabe». Mais Boitel, qui ne voyait aucune contradiction entre l'appellation de la méthode et son résultat, n'hésite pas à souligner les multiples avantages de son mode de conservation des cadavres. Avant tout, les coûts étaient moindres, surtout si l'on considère ceux de la pétrification de l'Italien Angelo Comi, nettement plus élevés. Selon lui, sa méthode donnait la possibilité à tout le monde d'avoir un mausolée pour l'éternité. En outre, elle n'exigeait «aucune des manipulations déplorables» (Boitel, s.d., p. 8, note) comme l'ouverture du corps, l'extraction des entrailles ou les injections aromatiques qui étaient pratiquées alors pour les embaumements ordinaires qui, selon lui, ne permettaient qu'une conservation temporaire et incertaine. Boitel souligne aussi les avantages au niveau sanitaire. Étant donnée l'enveloppe de pierre dans laquelle le cadavre est disposé, aucun élément lié à la putréfaction ne pouvait vicier l'air ou polluer les eaux: «ce serait un mode d'inhumation avantageux pour la salubrité de nos grandes villes» (Boitel, s.d., p. 3). Boitel a-t-il expérimenté sa méthode? N'appartenant pas à la profession médicale et n'ayant aucun lien avec le milieu scientifique, il est peu probable qu'il ait eu la possibilité de faire des expériences avec des cadavres. Son invention ne semble avoir laissé aucune trace dans la littérature scientifique ou professionnelle de l'époque ${ }^{9}$.

Si Boitel en publiant sa brochure s'adresse à un large public, Urban, quant à lui, envoie directement aux sénateurs et aux députés de la République française un mémoire (Urban, s.d.), imprimé à Reims et intitulé Mémoire sur l'andro-lithe, ou pétrification du corps humains après leur incinération. Ce texte ne porte aucune date, mais plusieurs éléments font penser à une date de publication entre 1875 et 1885 , année de création du crématorium du Père-Lachaise $\mathrm{e}^{10}$. Après avoir fait plusieurs expériences en incinérant le cadavre d'un chat, un squelette humain et le cœur d'un mouton et après avoir mélangé les cendres obtenues avec différents éléments comme le silicate de soude, l'argile, le kaolin ou le quartz, Urban dit avoir obtenu, à chaque fois, «des produits qui avaient toutes les qualités d'un grès très-dur, presque vitrifié et de couleurs variées »(Urban, s.d., p. 8 ). Son projet est de produire ce matériel avec les restes incinérés de cadavres humains et c'est pourquoi il appelle ce matériel «Andro-lithe», la «pierre humaine». 
On pouvait donner à cette pierre la forme désirée. Urban avait établi trois variantes principales. Des petites quantités de matériel pouvaient être coulées «dans une capsule d'or ou de platine de la forme qu'on voulait pour en faire une relique ou un bijou» (Urban, s.d., p. 7). Chaque forme proposée pouvait répondre aux désirs et aux possibilités économiques de chaque classe sociale. Pour les classes pauvres et moyennes, il propose les "pierres reliquaires » de forme cubique sur lesquelles il était possible de graver une inscription, de réaliser un portrait du défunt ou de placer une photo sur émail. Les personnes plus fortunées pouvaient opter pour un buste ou une statue, portant les traits de la personne disparue, recouvert d'émail, de bronze, d'argent ou d'or. Pour Urban, toutes les familles auraient donc eu la consolation «de pouvoir conserver pures et incorruptibles, les reliques de leurs parents qui par ce moyen » (Urban, s.d., p. 5) seraient passés à la postérité. Les pierres cubiques, les bustes, les statues auraient pu, sans aucune difficulté ou crainte, être placés en plein air, dans des chapelles funéraires, dans des musées de famille ou encore sous les voûtes d'une Église. Les pierres cubiques auraient aussi pu servir à la construction de monuments nationaux, de temples ou d'églises car, sur la face visible, on y trouvait le nom, les dates de la naissance et de la mort et la mention des qualités et vertus de la personne dont on voulait garder la mémoire. Devenu un matériau de construction ou une statue, l'Andro-lithe aurait permis de "conserver les restes mortels de ceux qui, par leur science, leur gloire et leurs vertus civiques, avaient bien mérité de la patrie, afin que les générations futures puissent voir, admirer et rendre hommage à leur image personnifiée avec leurs cendres vénérées » (Urban, s.d., p. 3). Avec son «innovation», Urban imaginait un monument qui absorbe, conserve et matérialise non seulement la mémoire et l'identité du défunt mais aussi son corps incinéré. La transformation en pierre des cendres des individus dont la famille ou les autorités voulaient conserver la mémoire était conçue comme un service public et comme «l'un des moyens des plus sûrs, des plus avantageux et des plus lucratifs pour aider la patrie à payer la dette énorme dont elle est accablée» (Urban, s.d., p. 4). L'État, en supervisant tout le processus, pouvait envisager la création d'un établissement céramique pour la confection de l'Androlithe dans chaque département, ou un seul au centre géographique de la France ou à Sèvres, siège de la manufacture nationale de porcelaine depuis le XVIII ${ }^{\mathrm{e}}$ siècle. Urban imaginait un réseau de crématoriums communaux: dans chaque crématorium, un à dix cadavres à la fois, mais séparément, pouvaient être incinérés. Les cendres à transformer en Andro-lithe, auraient été envoyées dans une boîte de fer bien fermée par le service postal à l'établissement céramique. Une fois la pierre obtenue, celle-ci aurait été transformée en sculpture par des artistes «de premier mérite», selon le goût et la fortune des parents du défunt: avec l'Andro-lithe, l'État aurait même pu «faire revivre et encourager les beaux-arts»(Urban, s.d., p. 10). Les tarifs, établis par l'État, auraient varié de 50000 à 100000 francs, selon la forme, la perfection et la décoration de la pierre. Une fois réalisée, chaque pierre aurait dû «être envoyée par une poste spéciale, à la mairie de chaque commune où les parents $d u$ défunt» (Urban, s.d., p. 13) pouvaient aller la réclamer.

D'après Urban, la production publique de l'Andro-lithe aurait été très avantageuse pour l'État qui n'aurait eu que des frais très minimes comparés «[aux] bénéfices immenses, car jamais il n'y [aurait eu] d'interruption dans la mortalité quotidienne»(Urban, s.d., p. 11). Les avantages économiques de «cette vaste entreprise» dirigée par l'État étaient présentés par des calculs qui envisageaient la transformation en pierre de la moitié des corps incinérés, environ 340000 pour toute la France. Quelle a été la réaction des députés et des sénateurs? Ont-ils débattu la question? Quelle diffusion a eu cet extravagant projet au-delà du document envoyé aux politiciens? Les recherches menées jusqu'ici ne permettent pas encore de le dire.

En Italie, à peu près à la même période, on trouve un important protagoniste de l'histoire de la pétrification humaine au $\mathrm{XIX}^{\mathrm{e}}$ siècle qui s'est consacré avec beaucoup de succès, et sans y voir aucune contradiction, tant à la conservation des cadavres qu'à leur destruction par incinération. Positiviste, républicain, impliqué dans le mouvement pour l'unité et l'indépendance italienne, Paolo Gorini (18131881) a étudié à Pavie pour s'établir ensuite à Lodi, en Lombardie, où il devient professeur de physique dans un lycée de la ville. Il s'occupe de géologie et de vulcanologie. Il publie divers ouvrages sur ces sujets, qui ne reçoivent pas un très bon accueil du milieu scientifique italien. Bientôt ses intérêts et ses recherches se tournent vers les techniques de la crémation et de la conservation du corps humain. (Gorini, 1881; Conti, 2002; Carli, 2005). Vers la moitié des années 1870, il met au point un four crématoire que la mairie de Lodi achètera pour le mettre en place (1877). Ses techniques seront aussi utilisées plus tard pour améliorer le grand crématorium de Milan. (Ginex et Selvafolta, 1996, p. 89).
Son intérêt pour l'origine des montagnes et pour les volcans avait amené Gorini à apprécier la perfection avec laquelle la nature conservait les plantes et les animaux en les transformant en fossiles. À partir des années 1840, il essaie lui-même de reproduire artificiellement le processus naturel de minéralisation des substances organiques. Contrairement à Segato et à Comi, et probablement grâce au soutien du milieu médical et hospitalier de la petite ville de Lodi, Gorini a pu concentrer ses expériences sur du matériel essentiellement humain. À côté des pièces anatomiques desséchées, parmi lesquelles plusieurs têtes avec leurs cheveux et leurs barbes, il réussira à pétrifier des mains, des pieds et des corps entiers. Sa renommée est telle qu'il est appelé en 1872 à conserver le corps de l'un des plus grands hommes politiques italiens, Giuseppe Mazzini, mais sans le pétrifier, chose qu'il arrivera à faire deux ans après avec le corps de l'écrivain Giuseppe Rovani (Luzzato, 2002; Carli 2005). Le succès de sa méthode est international. Dans un rapport sur l'évaluation des modes d'embaumement, par exemple, l'Académie de Médecine de Paris affirme que les pièces présentées par Gorini «sont d'une dureté comparable à celle de la pierre» et que visuellement elles ressemblent aux "plus belles exécutions en cire des parties du corps» (Poiseuille, Sucquet et Dupré, 1847, p. 30). Mais les membres de l'insigne institution française se plaignent du fait que Gorini n'a pas encore divulgué ses formules et qu'il garde son procédé secret (Poiseuille, Sucquet et Dupré, 1847, p. 30). Le «pétrificateur de Lodi » mourra en effet sans avoir dévoilé ses formules, même si subsistent quelques notes prises par son ami et médecin Luigi Rovida pendant la préparation du corps de Mazzini - processus qui, comme on l'a déjà dit, n'avait pas abouti à la pétrification (Carli, 2005). Le professeur Gorini a laissé une très riche collection anatomique qui, cataloguée dans les années 1980, est aujourd'hui conservée dans l'ancienne salle capitulaire du vieil hôpital de Lodi, auquel les héritiers l'avait donnée. Cette collection est composée d'environ 150 pièces ${ }^{11}$ dont du matériel préparé «à sec», entre autres 23 têtes humaines. Les résultats de son processus de pétrification peuvent être observés sur des organes, des membres supérieurs et inférieurs, des corps entiers d'enfants, dont un à genoux en prière, et deux corps humains entiers très bien conservés ${ }^{12}$. Comme le souligne Fausto Barbagli (2005), les pièces de Gorini n'avaient et n'auront jamais de fonction scientifique ou didactique; elles faisaient partie d'une sorte de cabinet de curiosités. Bien que ses découvertes eussent eu un grand écho en Italie et en Europe, elles ne 
suffirent pas à donner à Gorini une place dans une institution scientifique. Sa ville d'adoption fera cependant construire un monument public à sa mémoire, réussissant lentement à vaincre les résistances des milieux catholiques fortement opposés à la crémation.

Efisio Marini (1835-1900), autre grand protagoniste de l'histoire de la transformation en pierre des tissus, ne connaîtra pas cet honneur: après un succès international plus important que celui de Gorini, il perdra la raison et mourra dans la misère. Après ses études de médecine et d'histoire naturelle à Pise, il devient assistant au Musée d'histoire naturelle de Cagliari, en Sardaigne. Parallèlement à ses recherches en paléontologie et en photographie, il s'occupe de conservation des tissus en faisant ses expériences à l'École d'anatomie de l'Université de Cagliari et dans l'obituaire de la ville (Zedda et Serra, 2004 ; Zedda, 2005). Luigi Ferrara, dans une monographie dédiée à Marini, décrit les méthodes de ce dernier, qui étaient de trois types: une conservation «à l'état coriace transitoire», la pétrification et «le plus magnifique, une conservation permanente à l'état frais, avec flexibilité, souplesse et couleurs naturelles » (Ferrara, 1898 , p. 8$)^{13}$. En 1866 , il pétrifie le corps de l'historien Pietro Martini et l'événement suscita l'attention des journaux locaux de l'époque. Mais le peuple et même une partie du milieu cultivé de Cagliari se montra hostile ou violemment ironique envers ses découvertes. En 1867, Marini décide donc de quitter la Sardaigne pour aller à Paris où avait lieu l'Exposition universelle. Le public parisien, toujours assoiffé de nouveautés, accueillit très favorablement les découvertes du médecin italien. Sa capacité à redonner de la souplesse à un pied momifié semble avoir tellement impressionné Napoléon III que ce dernier chargea Nélaton de l'Académie de Médecine de Paris d'examiner attentivement les pièces pétrifiées par Marini (Ferrara, 1898). Nélaton était surtout intéressé par la capacité de Marini de rendre de la souplesse aux préparés desséchés. Après avoir soumis un pied momifié, il affirma que Marini avait réussi à rendre à la pièce une élasticité presque complète ${ }^{14}$. Dans ses articles, Luigi Ferrara évoque une table que Marini donna à l'Empereur, qui l'aurait lui-même confiée au Musée de l'Académie de Médecine ${ }^{15}$. La dédicace figure sur une plaque d'argent, accrochée à un pied humain, qui indique: «Table composée de différentes substances animales réduites à l'état de pierre par le docteur Efisio Marini dédié à S. M. l'Empereur [...]».

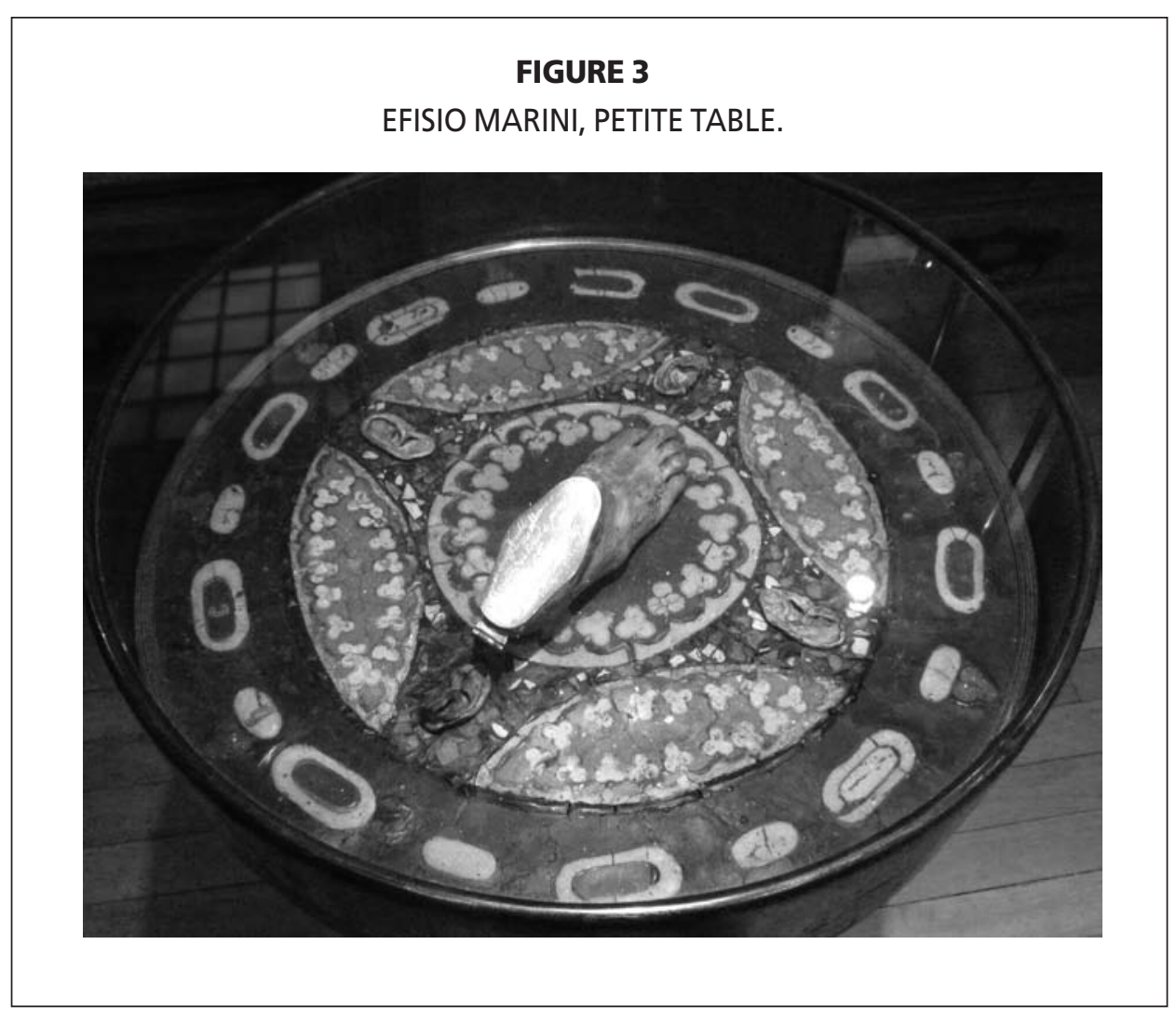

(C) Musée d'histoire de la médecine. Université Paris-Descartes

Après ce bref séjour parisien, Marini s'installe définitivement à Naples où il applique avec succès ses méthodes d'embaumement - et non pas de pétrification - à plusieurs membres décédés de la bourgeoisie locale. Le docteur de Cagliari serait aussi connu pour avoir pétrifié, lors d'une blessure, le sang de Garibaldi qui, après avoir été transformé en médaillon, fut donné au «héros des deux Mondes».

Marini tenta d'obtenir une reconnaissance formelle du monde scientifique italien afin d'avoir accès à un poste à l'université, mais cette reconnaissance n'arriva pas pour plusieurs raisons, parmi lesquelles son refus de rendre publiques ses formules. Au moment de l'Esposizione Industriale Italiana, à Milan, en 1881, Marini présenta officiellement les applications possibles de ses méthodes pour la production du cuir, pour l'étude de l'anatomie et de la chirurgie et même pour les enquêtes judiciaires. Mais rien de concret ne vint s'ajouter aux éloges, laissant Marini dans la solitude de son laboratoire, sans emploi fixe ni vrai soutien économique. Les articles publiés par Luigi Ferrara en 1898 en France, en Allemagne et en Italie suscitèrent l'attention de la presse internationale ${ }^{16}$, mais il était trop tard : l'art de conserver les corps développé par Marini resta une curiosité macabre, et ses secrets disparurent avec lui deux années plus tard. Seules quelques-unes de ses pièces ont sur- vécu et elles sont conservées au Musée anatomique de Naples ${ }^{17}$.

Un destin commun semble unir tous ces hommes qui ont consacré une partie plus ou moins importante de leur vie à développer différentes méthodes de pétrification du corps humain: l'échec au niveau de la diffusion et de l'application réelle. Les projets visionnaires de Boitel et d'Urban en sont probablement restés au stade de la conception. La transformation en pierre de tissus humains a suscité tantôt l'enthousiasme, tantôt une simple curiosité scientifique ou encore un intérêt macabre. Si l'art de Gerolamo Segato n'a pas pu se libérer d'une aura d'alchimie qui subsiste encore aujourd'hui pour une part, les résultats obtenus de "pétrificateurs » italiens qui l'ont suivi n'ont jamais connu une application dans le milieu scientifique, médical ou funéraire. À la pétrification, les anatomistes, les médecins, les chercheurs des sciences naturelles ont toujours préféré d'autres méthodes de conservation jugées plus efficaces et moins coûteuses, qui furent révolutionnées par la découverte du formaldéhyde (1867), par l'utilisation de la paraffine et par les techniques de réfrigération (voir Kleiss et Simonsberger, 1963). On pourrait penser que l'histoire de la pétrification des tissus humains se termine avec le $\mathrm{XIX}^{\mathrm{e}}$ siècle, mais on la retrouve plus tard parmi les recherches de Francesco Spirito 
(1885-1962), préparateur à l'Institut anatomique de Naples, puis gynécologue et recteur de l'Université de Sienne (Vannozzi, 2001). Donc, encore au $X X X^{\mathrm{e}}$ siècle, la pétrification n'a pas cessé de séduire les hommes de sciences, probablement parce qu'elle leur donne l'impression de vaincre la mort et d'imiter la nature.

\section{Bibliographie}

ACADÉMIE DES SCIENCES DE BOLOGNE (1840). Nuovi Annali delle Scienze Naturali, vol. 4, n ${ }^{\circ} 1$.

ALPAGO-NOVELLO, L. (1936). Tre documenti dell'archivio segreto vaticano riguardanti Girolamo Segato, Feltre, Stabilimento Tipografico Panfilio Castaldi.

ANTOLOGIA DI FIRENZE, vol. 44, nº 132. BARBAGLI, F. (2005). «La Wunderkamer di Paolo Gorini », dans A. CARLI (dir.), Storia di uno scienziato: collezione anatomica di Paolo Gorini, Azzano San Paolo, Bolis, p. 55-58.

BAYLE, D.C. (1873). L'embaumement dans les temps anciens et modernes. Suivi de l'exposé d'une méthode nouvelle sans incisions, Paris, A. Delahaye.

BERTLEY, J. (1985). The Story of Relics, Londres, Constable.

BOITEL, E.L.B.B. (s.d.). Nouveau mode de sépulture par la pétrification des corps, Paris, De Levé.

BOLLETTINO DELLE SCIENZE MEDICHE PUBBLICATO PER CURA DELLA SOCIETÀ MEDICO-CHIRURGICA DI BOLOGNA, VOL. 11, 1835.

BUStiCO, G. (1896). Girolamo Segato, Belluno, Premiata Tipografia Cavessago.

CHAMBERLAIN, A.T. et P.M. PARKER (2001). Earthly Remains. The History and Science of Preserved Human Bodies, Londres - New York, Oxford University Press.

CARLI, A. (dir.) (2005). Storia di uno scienziato: collezione anatomica di Paolo Gorini, Azzano San Paolo, Bolis.

COLIEZ, A. (1930). De la conservation artificielle des corps. Historique technique moderne des embaumements, Paris, Amédée Legrand.

COMET, C.-J.-B. (1846). Nouveau Procédé de conservation des cadavres et des préparations anatomiques par M. Paul Gorini, Paris, A. Bailly.

CONTI, F. (2002). «Paolo Gorini», dans Instituto dell'Enciclopedia Italiana, Dizionario Biografico degli Italiani, Rome, Instituto dell'Enciclopedia Italiana, p. 59-62.

COZZI, A. (1840). Ricerche sui metodi diversi fino ad ora adottati per effettuare le imbalsamazioni dei cadaveri e sulla riduzione delle sostanze organiche a solidita lapidea, Firenze, Pagani.

DAVIES, D. J. et L.H. MATES (dir.) (2005). Encyclopedia of Cremation, Aldershot, Ashgate.
D.G.R. (1839). Intorno all'artificiale solidificazione lapidea delle parti organico-animali ed al conservare $i$ vegetabili. Metodo scoperto dal Signor Angelo Comi romano, chirurgo [...], Discorso del prof. D.G.R., Pise, Stamperia Peraccini.

FAVOLE, A. (2003). Resti di Umanità : vita sociale del corpo dopo la morte, Rome, Laterza.

FERRARA, L. (1898). La sopravvivenza del corpo e le scoperte di Efisio Marini, Trani, Vecchi.

GANNAL, J.N. (1848). Rapport de l'Académie des Sciences sur les procédés d'embaumement de M. Gannal, Séance du 24 mars 1848, Paris, Impr. de Le Normant.

GANNAL, J. N. (1838). Histoire des embaumements et de la préparation des pièces d'anatomie normale, d'anatomie pathologique et d'histoire naturelle; suivie de procédés nouveaux, Paris, Ferra.

GINEX, G. et O. SELVAFOLTA (1996). Il Cimitero Monumentale di Milano. Guida storico-artistica, Milan, Silvana Editrice.

GORINI, P. (1881). Autobiografia, Rome, Dossi-Perelli-Levi.

GRILLETTO, R (1986). «Imbalsamazione e "pietrificazione " ", dans A. SILIOTTI (dir.), Belluno e l'Egitto, Celebrazioni in onore di Girolamo Segato nel $150^{\circ}$ anniversario della morte, Vérone, Rassegna internazionale di cinematografia archeologica, p. 52-54.

GRILLETTO, R (1996). Il mistero delle mummie. Dall'antichità ai nostri giorni attraverso il tempo e lo spazio, Rome, Newton Compton Editori.

HAUSSMANN, G.E. (2000). Mémoires du Baron Haussmann, édition établie par Françoise CHOAY, Paris, Seuil.

KLEISS, E. et P. SIMONSBERGER (1963). La parafinizacion como metodo morfologico, Merida, Universidad de los Andes Facultad de Medicina.

LASKOWSKI, S. (1886). L'embaumement, la conservation des sujets et les préparations anatomiques, Genève - Lyon, H. Georg.

LISTA, L. (2005). Raimondo di Sangro: il principe dei veli di pietra, Foggia, Bastogi.

LUZZATO, S. (2002). La mummia della repubblica: storia di Mazzini imbalsamato, 1872-1946, Milan, Rizzoli.

MACCIONI, A. (1996 [1992-1993]). «Efisio Marini e la conquista dell'eternità », Studi Sardi, vol. 30, p. 680-687.

MAFFIOLI, M. et P. RUSCHI (1986). Santa Croce nell'800, Firenze, Fratelli Alinari Editrice.

MANDRESSI, R. (2003). Le regard de l'anatomiste, Dissections et inventions du corps en Occident, Paris, Seuil.

MAYER, R.G. (1990). Embalming : History, Theory and Practice, Stanford, Appleton and Lange.
MAZZONI, L. (1938). Un viaggiatore toscano dei primi dell'800: lettere di G. Mazzoni riassunte e ordinate da Luigi Mazzoni, Firenze, Tip. F.lli Stianti.

PELLEGRINI, G. (1836). Della artificiale riduzione a solidità lapidea e inalterabilità degli animali scoperta da Girolamo Segato: relazione dell'avvocato Giuseppe Pellegrini con note ed aggiunte di prose e poesie, Firenze, V. Batelli e figli.

PIERI, G. (1936). Girolamo Segato (17921836), Belluno, Istituto Veneto di Arti Grafiche.

POCCHIESA, I. et M. FORNARO (1992) Girolamo Segato esploratore dell'ignoto. Scienziato, viaggiatore, cartografo, Belluno, Media Diffusion.

POISEUILLE, J.-L.-M., P. SUCQUET et DUPRÉ (1847). Rapports sur divers modes d'embaumement présentés par MM. Dupré, Gannal et Sucquet [...], Paris, J.-B. Baillière.

RICCI, G. (1998). Il principe e la morte: corpo, cuore, effigie nel Rinascimento, Bologna, Il Mulino.

RIVIÈRE, L.-F. et A. ROCHE (1877). Des embaumements, De la conservation et de la pétrification des corps, Paris, de Bernard.

SEGATO, G. (1831). Antologia di Firenze, vol. $44, \mathrm{n}^{\circ} 132$

SILIOTTI, A. (dir.) (1986). Belluno $e$ l'Egitto, Celebrazioni in onore di Girolamo Segato nel $150^{\circ}$ anniversario della morte, Vérone, Rassegna internazionale di cinematografia archeologica.

SUCQUET, J.-P. (1872). De l'embaumement chez les anciens et chez les modernes et des conservations d'anatomie normale et pathologique, Aurillac, Impr. A. Pinard.

TERRIBILE WIEL MARIN, T. et C. CORRAIN (1986). "Pratiche imbalsamatorie in Europa», Pathologica, vol. 78, p. 107-118.

URBAN, (s.n.) (s.d.). Mémoire sur l'androlithe ou pétrification des corps humains après leur incinération, Reims, Imprimerie de l'Indépendant Rémois.

VANNOZZI, F. (2001). «I pezzi pietrificati dei fisiocratici di Siena ", Atti dell'Accademia delle Scienze di Siena, $\mathrm{n}^{\circ} 17$, suppl. p. 1-13.

VIDOR, G.M. (2005). "Mummificazione e Imbalsambazione», Études thanatologiques - Studi Tanatologici - Thanatological Studies, $\mathrm{n}^{\circ} 1$, p. 207-214

ZANON, B. (1839). Processo di solidificazione dei corpi animali, Memoria di Bartolommeo Zanon, Belluno, Deliberali.

ZEDDA, C. et L. SERRA (dir.) (2004). Il Pietrificatore Efisio Marini (Cagliari 1835 Napoli 1900), Elmas, Grafiche Sainas.

ZEDDA, C. (2005). «Efisio Marini e Paolo Gorini : due personaggi a confronto", dans A. CARLI (dir.), Storia di uno scienziato: collezione anatomica di Paolo Gorini, Azzano San Paolo, Bolis, p. 81-88. 


\section{Notes}

1. À ne pas confondre avec son célèbre cousin, le peintre Jean Honoré Fragonard.

2. Aujourd'hui conservé avec d'autres écorchés au Musée Fragonard de l'École nationale vétérinaire d'Alfort.

3. Encore conservées dans la Cappella San Severo, aujourd'hui transformée en musée: voir <http://www.museosansevero.it/>.

4. Voir le compte-rendu de la Seduta delli 19 maggio 1835 dans le Bollettino delle Scienze Mediche pubblicato per cura della società medico-chirurgica di Bologna, vol. 11, 1835, p. 361-364.

5. Cette table, de proprieté du Museo Galileo de Florence, est conservée, ainsi que d'autres pièces préparées par Segato, au Museo Anatomico Fiorentino Dipartimento di Anatomia Istologia e Medicina Legale de l’Université de Florence.

6. Au cours du XIX ${ }^{\mathrm{e}}$ siècle, Florence connaît un grand développement intellectuel, culturel et artistique. L'église de Santa Croce, au centre de grands projets de restauration, se transformera au cours du siècle en une sorte de Panthéon italien. À côté de vrais tombeaux de représentants de la culture florentine et italienne de l'époque, des magnifiques monuments honoraient les contemporains ensevelis ailleurs comme Rossini, mais aussi les grands du passé comme Dante, Machiavel et Donatello. Le fait d'enterrer Segato à Santa Croce constituait une reconnaissance de la valeur de ses activités d'explorateur et de "pétrificateur» (voir Maffioli et Ruschi, 1986).

7. «Emulatore di Segato » : ce qualificatif accompagne le portrait du professeur Angelo Comi dans D.G.R., 1839.

8. Voir Académie des sciences de Bologne, 1840, p. $138-140$.

9. La pétrification continue par contre à inspirer la littérature fantastique française du $\mathrm{XXI}^{\mathrm{e}}$ siècle. Ainsi, un cadavre de pierre découvert au cimetière du Père-Lachaise et datant $\mathrm{du} \mathrm{XIX}^{\mathrm{e}}$ siècle est au cœur du scénario de Ontophage. Pierres de Brume, bande dessinée publiée en 2009 par l'éditeur Emmanuel Proust.

10. L'auteur, à la page 11, cite le nombre des décès en 1874 et sa façon d'approcher la question de la crémation fait dater l'ouvrage avant la création en 1885 du crématorium du PèreLachaise.

11. Une liste des pièces exposées avec des photographies est publiée dans Carli, 2005.

12. La collection fait partie aujourd'hui du circuit muséal de la ville et elle est accessible au public. Voir <http://www.museilodi.it/>.

13. Ferrara, L. La sopravvivenza del corpo e le scoperte di Efisio Marini. Vecchi, Trani 1898. Cette monographie est la traduction, faite par l'auteur lui-même, de ses deux articles sur Efisio Marini parus l'un dans la Revue des Revues du $1^{\text {er }}$ août 1898 et l'autre dans la Berliner Illustrerte Zeitung du 2 octobre 1898.
14. Il le déclara dans Les Mondes, revue hebdomadaire des sciences de leurs applications [...] vol. 16, no 11,1868 , p. 429. L'information est donnée par Ferrara, 1898, p. 10.

15. Cette table est conservée au Musée d'histoire de la médecine de l'Université Paris-Descartes à Paris.

16. L'auteur donne une liste détaillée des articles parus dans d'importants journaux et revues de l'époque (Ferrara, 1898; voir Appendice, p. 43-58).

17. Corrado Zedda et Luigi Serra ont créé un site internet dédié à Efisio Marini et même une bande dessinée sur sa vie (voir <http://www. efisiomarini.info $>$ ). L'écrivain Giorgio Todde a aussi fait de Efisio Marini un personnage dans quelques-uns de ses romans, dont $L o$ Stato delle Anime, 2002, et Paura e Carne, 2003. 